\title{
Survival Analysis in Patients With Preserved Left Ventricular Function and Standard Indications for Permanent Cardiac Pacing Randomized to Right Ventricular Apical or Septal Outflow Tract Pacing
}

\author{
Alicja Dąbrowska-Kugacka, MD; Ewa Lewicka-Nowak, MD; Sebastian Tybura, MD; \\ Rajmund Wilczek, MD; Justyna Staniewicz, MD; \\ Paweł Zagożdżon, MD*; Anna Faran, MD; Dariusz Kozłowski, MD; \\ Grzegorz Raczak, PhD; Grażyna Świątecka, PhD
}

\begin{abstract}
Background: Optimal right ventricular (RV) pacing site in patients referred for permanent cardiac pacing remains controversial. A prospective randomized trial was done to compare long-term effect of permanent RV apex (RVA) vs RV outflow tract (RVOT) pacing on the all-cause and cardiovascular mortality.

Methods and Results: A total of 122 consecutive patients (70 men, $69 \pm 11$ years), with standard pacing indications were randomized to RVA (66 patients) or RVOT (56 patients) ventricular lead placement. After the 10year follow-up period the mortality data were summarized on the basis of an intention-to-treat analysis. During the long-term follow-up, 31 patients from the RVA group died vs 24 patients in the RVOT group (hazard ratio (HR), $0.96 ; 95 \%$ confidence interval (CI), $0.57-1.65 ; \mathrm{P}=0.89$ ). There were 10 cardiovascular deaths in the RVA and 12 in the RVOT group (HR, 1.04; 95\% CI, 0.45-2.41; P=0.93). There were no differences in the all-cause or cardiovascular mortality between the pacing sites after adjustment for age, gender, arterial hypertension, atrial fibrillation, New York Heart Association class and left ventricular end-diastolic diameter.
\end{abstract}

Conclusions: The RVOT provides no additional benefit in terms of long-term survival over RVA pacing. (Circ J 2009; 73: 1812-1819)

Key Words: Cardiovascular death; Permanent cardiac pacing; Right ventricular apical pacing; Right ventricular outflow tract pacing; Survival

$\mathbf{T}$ The traditional site for ventricular lead placement-the right ventricular apex (RVA), produces an abnormal pattern of ventricular depolarization, and there is growing evidence that pacing from this site is associated with detrimental functional and structural changes in the heart which might lead to an adverse clinical outcome. ${ }^{1-3}$ These observations have increased interest in pacing at sites alternative to the RVA, mainly in the area of the right ventricular outflow tract (RVOT). It has been hypothesized that pacing in the RVOT, owing to its proximity to the His-bundle, results in a more physiological depolarization pattern and better hemodynamics and might reduce detrimental effects of long-term ventricular pacing. Results of a meta-analysis comparing RVA to RVOT pacing have suggested acute benefit from pacing at the RVOT. ${ }^{4}$ However, poor definition of the outflow tract area and the non-randomized character of most trials confound the data. Longterm evaluation of the effects of chronic pacing are limited, although some mid-term observations show equivalency between apical and outflow tract pacing. ${ }^{5,6}$ Other studies indicate that, in contrast to RVA, RVOT pacing preserves left ventricular (LV) function. ${ }^{7}$ However, it is still not clear whether RVOT pacing provides better long-term outcome than RVA pacing.

In this study we investigated RVOT pacing in comparison to RVA pacing in patients with standard indications for permanent ventricular pacing and preserved LV systolic function to determine whether RVOT pacing would provide better all-cause and cardiovascular survival.

\section{Methods}

The present study was a single-center randomized study performed in a tertiary care university hospital. The first patient was enrolled on $7^{\text {th }}$ September 1995 and the last patient on $24^{\text {th }}$ November 1997. After the 10-year follow-up visit the long-term survival in the studied population was evaluated.

\section{Patients}

Patients were eligible for the study if they were at least 21 years old, had preserved LV ejection fraction (LVEF)

(Received March 1, 2009; accepted June 7, 2009; released online August 19, 2009)

Department of Cardiology and Electrotherapy, *Department of Hygiene and Epidemiology, Medical University of Gdańsk, Gdańsk, Poland

Mailing address: Alicja Dąbrowska-Kugacka, MD, Department of Cardiology and Electrotherapy, Medical University of Gdańsk, Dębinki 7, 80-211 Gdańsk, Poland. E-mail: adabrowska@amg.gda.pl

All rights are reserved to the Japanese Circulation Society. For permissions, please e-mail: cj@j-circ.or.jp 
$>40 \%$, and were referred for initial, non-urgent implantation of a VDD, DDD or VVI/R pacemaker according to standard indications for permanent cardiac pacing. Patients participating in the study had to be willing to attend to the follow-up control visits in the outpatient clinic.

\section{Randomization Procedure}

Before randomization all patients gave their written consent to participation in the study. The randomization process was performed on the basis of numbered containers. The interventions (RVA or RVOT ventricular lead placement in $1: 1$ proportion) were sealed in 150 sequentially numbered opaque identical envelopes. On the basis of randomization the intention-to-treat survival analysis was performed. All implantations were performed by the most experienced operator in the center (RW).

The study protocol complies with the Declaration of Helsinki, was approved by the local ethical committee, and all patients gave their written informed consent to pacemaker implantation and participation in the study.

\section{Pacemaker Implantation Procedure}

The atrial lead was implanted in the right atrial appendage. The RVOT lead was advanced through the tricuspid valve into the right ventricle (RV) and further into the pulmonary artery. It was then withdrawn slowly until the tip of the electrode dropped below the pulmonary valve, far enough to position the tip against the interventricular septum, approximately two-thirds of distance from the apex to the pulmonary valve in the anterior-posterior view, and toward the septum (high septum) in the left anterior oblique view. Localization of the electrode was verified during the implantation with fluoroscopy. A left lateral oblique chest X-ray demonstrating ventricular lead position in the RVOT is presented in Figure 1. The RVA lead was placed typically in the RV apex. In all patients passive fixation leads were used at all pacing sites: models SL 60 electrodes in all VDD pacemakers; SX 60, SX 53 or TIR 60 at ventricular and TIJ 53 at atrial sites (Biotronik $\mathrm{GmbH}$, Berlin, Germany). The following models of pacemakers were implanted: Dromos SL/SR, Ergos 03, Physios 01, Pikos 01 (Biotronik GmbH). In all patients a 12-lead surface electrocardiogram (ECG) was performed at hospital discharge (3-5 days after pacemaker implantation) at $25 \mathrm{~mm} / \mathrm{s}$ paper speed with a gain of $10 \mathrm{~mm} / \mathrm{mV}$. Left bundle branch block morphology with an inferior axis in the frontal plane during forced ventricular pacing indicated the RVOT as the pacing site.

\section{Follow-up}

The pacemakers were programmed with a basic rate between 65 and 85 beats/min, and in patients with chronotropic incompetence the rate-response function was switched on. The atrio-ventricular (AV) delay of the VDD and DDD pacemakers was initially programmed to the standard value of $160 \pm 30 \mathrm{~ms}$. Control visits were performed in the outpatient clinic four times during the first year after implantation and at least once a year thereafter. During the postimplantation period pharmacological treatment has been prescribed individually on the basis of the clinical status of the patients. In order to verify the long-term ventricular lead stability and to compare the percentage of ventricular pacing between the groups, these data were retrieved from the ECG and pacemaker data logs obtained during the last two control visits in the outpatient clinic.

In order to complete survival data of the initial study

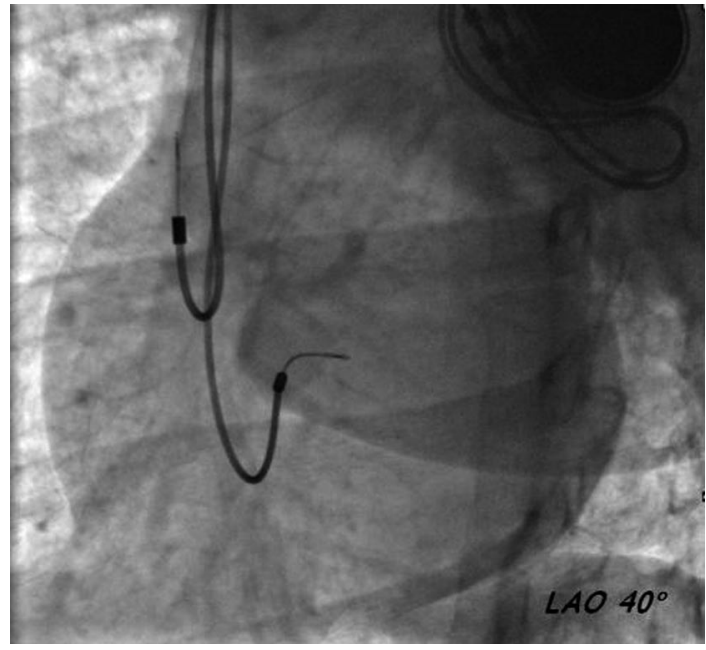

Figure 1. Left lateral oblique (LAO) $40^{\circ}$ chest X-ray film demonstrating ventricular pacing lead position in the right ventricular outflow tract.

population, data concerning the date and origin of death were retrieved from the outpatient clinic database and local statistical office after the scheduled 10-year control visit. Cardiovascular death was defined as death due to cardiac arrhythmia, death from non-arrhythmic cardiac causes or death from non-cardiac vascular causes.

\section{Statistical Methods}

Data are presented as mean with standard deviations or median with ranges where appropriate. A Fisher exact test was used to analyze differences between categorical variables. For normally distributed continuous variables the Student's t-test was applied. If variables did not follow normal distribution the Mann-Whitney test was performed for comparisons between different treatment groups. Comparison was made according to the intention-to-treat basis.

The Kaplan-Meier method was used to calculate survival rates and cardiovascular fatal event-free rates, and results were compared by the log-rank test. The Cox proportional hazard regression method was applied to identify variables that independently predicted overall and cardiovascular mortality. The New York Heart Association (NYHA) class was coded as a dummy variable. Predictors with univariate $\mathrm{P}$-value $\leq 0.2$ were included in the multivariate Cox regression model, although in the case of co-linearity between two or more variables only one of them was included in the multivariate model. In the regression analysis, co-linearity of two variables means that a strong correlation exists between them, making it difficult or impossible to estimate their individual regression coefficients reliably. For that reason LVEF showing co-linearity with the LV end-diastolic diameter (LVEDD) was not taken into consideration in multivariate analysis. Relative risk was expressed as a hazard ratio (HR) with a $95 \%$ confidence interval (CI). ${ }^{8}$

STATA software (version 9.2, StatCorp) was used to calculate statistics. A P-value of $<0.05$ was considered statistically significant.

\section{Results}

Baseline Characteristics

During the randomization period ( $7^{\text {th }}$ September $1995-24^{\text {th }}$ 


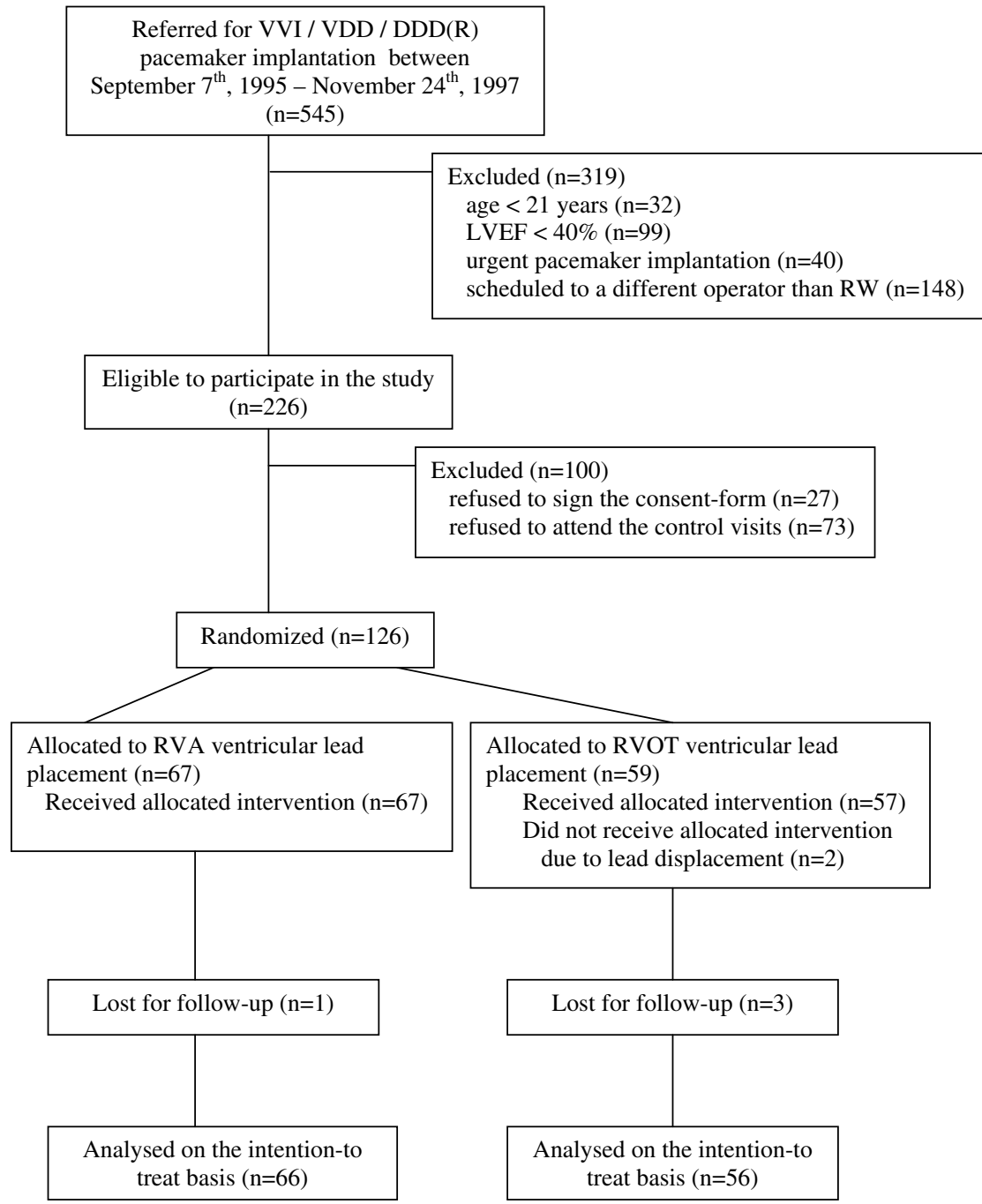

Figure 2. Diagram showing the flow of participants through each stage of the study. RVA, right ventricular apical; RVOT, right ventricular outflow tract.

Table 1. Baseline Characteristics of the Study Group According to Intention-to-Treat Analysis

\begin{tabular}{lccc}
\hline & RVA pacing $(\mathrm{N}=66)$ & RVOT pacing $(\mathrm{N}=56)$ & P value \\
\hline Age (years) & $72(63-77)$ & $72(62-76)$ & 0.48 \\
Male gender & $40(54 \%)$ & $30(62 \%)$ & 0.46 \\
Body mass index $\left(\mathrm{kg} / \mathrm{m}^{2}\right)$ & $26(24-28)$ & $28(24-30)$ & 0.31 \\
Hypertension & $24(36 \%)$ & $26(46 \%)$ & 0.27 \\
Coronary artery disease & $19(34 \%)$ & $14(21 \%)$ & 0.15 \\
Previous myocardial infarction & $3(5 \%)$ & $8(14 \%)$ & 0.11 \\
Chronic atrial fibrillation & $13(20 \%)$ & $18(32 \%)$ & 0.15 \\
Diabetes mellitus & $7(11 \%)$ & $6(11 \%)$ & 1.00 \\
Heart failure symptoms at implant: & $13(21 \%)$ & $11(20 \%)$ & \\
NYHA I & $23(38 \%)$ & $24(43 \%)$ & 0.25 \\
NYHA II & $5(8 \%)$ & $10(18 \%)$ & 0.07 \\
NYHA III & $53(50-57)$ & $51(47-54)$ & 0.37 \\
LVEDD (mm) & $55(45-60)$ & $54(45-60)$ & 0.87 \\
LVEF (\%) & $18 / 22 / 26$ & $15 / 21 / 20$ & 0.09 \\
VVI/VDD/DDD pacemakers & $166 \pm 21$ & $159 \pm 20$ & \\
QRS duration (ms) & & & \\
\hline
\end{tabular}

RVA, right ventricular apical; RVOT, right ventricular outflow tract; NYHA, New York Heart Association heart failure class; LVEDD, left ventricular end-diastolic diameter; LVEF, left ventricular ejection fraction.

November, 1997) 545 patients were referred to our department for VVI, VDD or DDD/R pacemaker implantation, of which 226 were eligible to participate in the study (age over 21, LVEF $>40 \%$, non-urgent pacemaker implantation scheduled to be performed by RW). One hundred patients refused to sign the study consent-form or to attend the control visits in our outpatient department. A total of 126 patients were randomized to participation in the study (67 patients to the RVA and 59 to the RVOT group), however during the follow-up 1 patient from the RVA and 3 from 

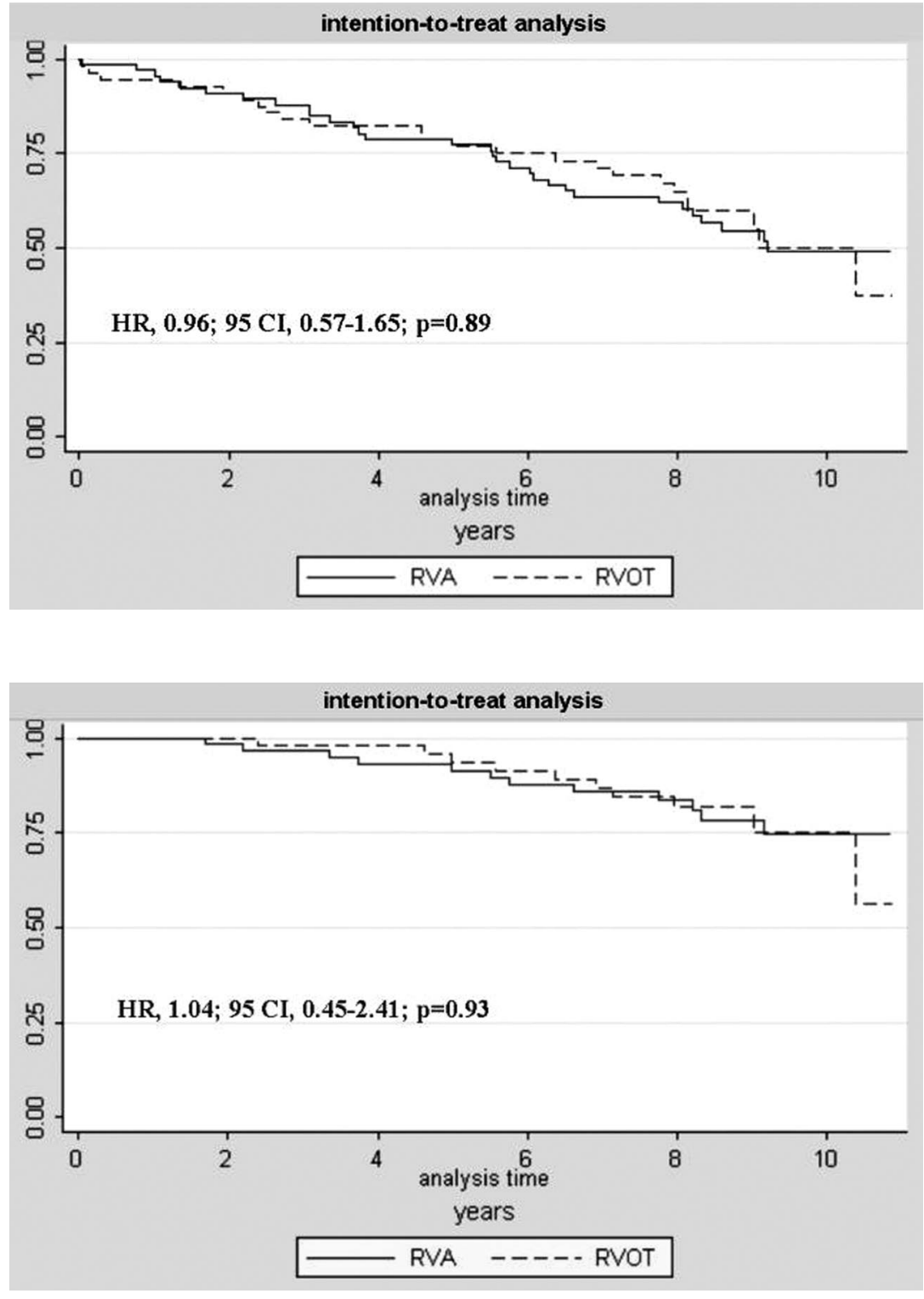

Figure 3. Kaplan-Meier survival curves in patients assigned to right ventricular apical (RVA) or septal right ventricular outflow tract (RVOT) pacing. HR, hazard ratio; CI, confidence interval.
Figure 4. Patients free from cardiovascular death (Kaplan-Meier curves): comparison between patients assigned to right ventricular apical (RVA) or septal right ventricular outflow tract (RVOT) pacing. HR, hazard ratio; CI, confidence interval. the RVOT group were lost for follow-up. The flow diagram is presented in Figure 2.

The final analysis was performed in 122 patients (mean age $69 \pm 11$ years, 70 men). At the time of implantation the majority of patients $(70 \%)$ demonstrated symptoms of heart failure, $41 \%$ had arterial hypertension, $27 \%$ coronary artery disease, $9 \%$ history of myocardial infarction, $25 \%$ chronic atrial fibrillation, and $11 \%$ diabetes mellitus. The mean LVEDD and LVEF were within the normal range $(52 \pm 5 \mathrm{~mm}$ and $55 \pm 6 \%$, respectively).

The indication for pacemaker implantation was symptomatic sinus node disease with low Wenkcebach point ( $<110$ beats/min) or bundle branch block in $14 \%$ of patients, chronic atrial fibrillation with bradycardia in $20 \%$ and second and/or third degree AV block in $66 \%$ of pacemaker recipients. A total of 33 patients were assigned to VVI pacemakers, 43 to VDD and 46 to $\mathrm{DDD} / \mathrm{R}$. None of the patients was implanted with a cardioverter-defibrillator.

\section{Comparison Between the Groups}

According to the intention-to-treat analysis 66 patients were classified to the RVA group, while 56 to the RVOT group. Comparison of the baseline characteristics of the study groups is presented in Table 1. There were no major differences between the groups, except for the LVEDD, which tended to be smaller in the RVOT patients.

The pacemaker mode assigned (VVI/VDD/DDD/R) did not differ between the groups. All patients allocated to RVA lead placement received the therapy, however, in the early post-implantation period, a displacement of the ventricular lead from the RVOT to RVA was observed in 2 patients initially randomized to the RVOT group. There was a trend to a shorter QRS width during RVOT pacing (RVOT: $159 \pm 20 \mathrm{~ms}$ vs RVA: $166 \pm 21 \mathrm{~ms} ; \mathrm{P}=0.09$ ).

There were no differences in complication incidence between both groups. Only 3 minor complications occurred: pneumothorax (1 patient in the RVA and 1 RVOT group) and 1 minor hematoma (RVA patient). The duration of hospitalization time due to the implantation did not differ between the groups $(4.37 \pm 1.3$ days vs $4.55 \pm 1.44$ days; $\mathrm{P}=$ 0.47 in the RVA and RVOT group, respectively). 
Table 2. Predictors of (a) All-Cause Mortality, (b) Cardiovascular Mortality Based on the Intention-to-Treat Analysis Evaluated by the Cox Proportional Hazard Regression Model

\begin{tabular}{|c|c|c|c|c|c|c|c|c|}
\hline & \multicolumn{4}{|c|}{ (a) All-cause death } & \multicolumn{4}{|c|}{ (b) Cardiovascular mortality } \\
\hline & \multicolumn{2}{|c|}{ Univariate analysis } & \multicolumn{2}{|c|}{ Multivariate analysis } & \multicolumn{2}{|c|}{ Univariate analysis } & \multicolumn{2}{|c|}{ Multivariate analysis } \\
\hline & $\begin{array}{c}\mathrm{HR} \\
(95 \% \mathrm{CI}) \\
\end{array}$ & $P$ value & $\begin{array}{c}\mathrm{HR} \\
(95 \% \mathrm{CI}) \\
\end{array}$ & $P$ value & $\begin{array}{c}\mathrm{HR} \\
(95 \% \mathrm{CI}) \\
\end{array}$ & $P$ value & $\begin{array}{c}\mathrm{HR} \\
(95 \% \mathrm{CI}) \\
\end{array}$ & $P$ value \\
\hline Ventricular pacing site (RVOT vs RVA) & $\begin{array}{c}0.96 \\
(0.57-1.65)\end{array}$ & 0.89 & $\begin{array}{c}1.41 \\
(0.76-2.64)\end{array}$ & 0.28 & $\begin{array}{c}1.04 \\
(0.45-2.41)\end{array}$ & 0.93 & $\begin{array}{c}1.66 \\
(0.61-4.52)\end{array}$ & 0.32 \\
\hline Gender (M vs F) & $\begin{array}{c}2.41 \\
(1.33-4.38)^{*}\end{array}$ & $0.00 *$ & $\begin{array}{c}4.78 \\
(2.10-10.89)^{*}\end{array}$ & $0.00 *$ & $\begin{array}{c}2.59 \\
(1.01-6.64)^{*}\end{array}$ & $0.05^{*}$ & $\begin{array}{c}5.37 \\
(1.66-17.40)^{*}\end{array}$ & $0.01^{*}$ \\
\hline Age at implantation & $\begin{array}{c}1.08 \\
(1.04-1.11)^{*}\end{array}$ & $0.00 *$ & $\begin{array}{c}1.10 \\
(1.05-1.15)^{*}\end{array}$ & $0.00 *$ & $\begin{array}{c}1.07 \\
(1.02-1.13)^{*}\end{array}$ & $0.01 *$ & $\begin{array}{c}1.12 \\
(1.04-1.21)^{*}\end{array}$ & $0.00 *$ \\
\hline Coronary artery disease & $\begin{array}{c}1.35 \\
(0.76-2.40)\end{array}$ & 0.31 & & & $\begin{array}{c}1.16 \\
(0.45-2.98)\end{array}$ & 0.76 & & \\
\hline History of myocardial infarction & $\begin{array}{c}1.40 \\
(0.60-3.28)\end{array}$ & 0.44 & & & $\begin{array}{c}1.80 \\
(0.53-6.12)\end{array}$ & 0.35 & & \\
\hline Arterial hypertension & $\begin{array}{c}0.65 \\
(0.37-1.15)\end{array}$ & 0.14 & $\begin{array}{c}1.02 \\
(0.50-2.09)\end{array}$ & 0.95 & $\begin{array}{c}0.75 \\
(0.31-1.78)\end{array}$ & 0.51 & & \\
\hline Atrial fibrillation & $\begin{array}{c}1.25 \\
(0.70-2.25)\end{array}$ & 0.45 & & & $\begin{array}{c}1.79 \\
(0.75-4.29)\end{array}$ & 0.19 & $\begin{array}{c}1.94 \\
(0.66-5.65)\end{array}$ & 0.23 \\
\hline Pacing mode (VVI vs DDD) & $\begin{array}{c}1.20 \\
(0.67-2.15)\end{array}$ & 0.54 & & & $\begin{array}{c}1.77 \\
(0.74-4.28)\end{array}$ & 0.20 & & \\
\hline $\begin{array}{l}\text { Heart failure symptoms at implantation: } \\
\text { None }\end{array}$ & 1.0 & & 1.0 & & 1.0 & & 1.0 & \\
\hline NYHA I & $\begin{array}{c}1.02 \\
(0.50-2.08)\end{array}$ & 0.96 & $\begin{array}{c}0.59 \\
(0.23-1.53)\end{array}$ & 0.28 & $\begin{array}{c}0.55 \\
(0.14-2.22)\end{array}$ & 0.40 & $\begin{array}{c}0.33 \\
(0.07-1.64)\end{array}$ & 0.18 \\
\hline NYHA II & $\begin{array}{c}1.09 \\
(0.48-2.48)\end{array}$ & 0.83 & $\begin{array}{c}0.86 \\
(0.40-1.81)\end{array}$ & 0.95 & $\begin{array}{c}0.70 \\
(0.24-2.09)\end{array}$ & 0.53 & $\begin{array}{c}0.53 \\
(0.15-1.82)\end{array}$ & 0.31 \\
\hline NYHA III & $\begin{array}{c}2.52 \\
(1.11-5.72)^{*}\end{array}$ & $0.03 *$ & $\begin{array}{c}1.95 \\
(0.78-4.91)\end{array}$ & 0.15 & $\begin{array}{c}3.09 \\
(0.99-9.67)^{*}\end{array}$ & $0.05^{*}$ & $\begin{array}{c}4.19 \\
(0.99-17.66)^{*}\end{array}$ & $0.05 *$ \\
\hline LVEDD & $\begin{array}{c}1.07 \\
(1.03-1.11)^{*}\end{array}$ & $0.00 *$ & $\begin{array}{c}1.03 \\
(0.99-1.08)\end{array}$ & 0.16 & $\begin{array}{c}1.08 \\
(1.01-1.16)^{*}\end{array}$ & $0.02 *$ & $\begin{array}{c}1.04 \\
(0.97-1.12)\end{array}$ & 0.26 \\
\hline LVEF & $\begin{array}{c}0.98 \\
(0.95-1.00)\end{array}$ & 0.10 & $\begin{array}{l}\text { Not included due } \\
\text { to co-linearity } \\
\text { with LVEDD }\end{array}$ & & $\begin{array}{c}0.95 \\
(0.91-0.99)^{*}\end{array}$ & $0.01 *$ & $\begin{array}{l}\text { Not included due } \\
\text { to co-linearity } \\
\text { with LVEDD }\end{array}$ & \\
\hline
\end{tabular}

HR, hazard ratio; CI, confidence interval. Other abbreviations see in Table 1.

\section{The Last Follow-up Visit}

After 10 years of the follow-up period, 57 patients out of the initial study group of 122 were still under control in the outpatient clinic (30 RVA and 27 RVOT patients). Fiftyfive patients died, and 10 moved from our region, but they were alive, and still in phone contact with the outpatient clinic. We were unable to retrieve survival data of 4 patients ( 1 from the RVA and 3 from the RVOT group). The mean duration of follow-up was $7 \pm 2.9$ years, and in the survivors $9.7 \pm 0.6$ years.

A comparison of the ECGs obtained in patients at hospital discharge and during the last control visit in the outpatient clinic revealed an unchanged QRS axis and morphology, which provided evidence for stable long-term ventricular lead position. Data obtained from the pacemaker data logs showed no difference in the percentage of ventricular pacing between the groups: $95 \pm 11 \%$ and $94 \pm$ $19 \%(\mathrm{P}=0.83)$ in the RVA and RVOT group, respectively. The pacing rate did not differ: $70.5 \pm 6.5$ beats/min vs $71.6 \pm$ 5.8 beats/min; $\mathrm{P}=0.4$ in the RVA and RVOT group, respectively. As almost all patients presented with normal LVEF the AV delay was not routinely programmed under echoDoppler. The echocardiographic examinations were performed on routine basis during the follow-up control visits, and only if the echocardiographer noticed important AV dyssynchrony (LV diastolic filling time $<40 \%$ of the RR interval) a setting under echo-Doppler was performed (in 4 patients from RVA and 3 from RVOT group). The mean AV delay during the last control visit was $145 \pm 25$ vs $149 \pm$ $23 \mathrm{~ms} ; \mathrm{P}=0.67$ in the RVA and RVOT group, respectively.

\section{Survival Analysis}

Data obtained from the outpatient clinical database and the statistical office revealed that 55 patients had died and that of these deaths, 22 had been from a cardiovascular cause. The mean time to death was $6.4 \pm 3$ years, and to cardiovascular death $6 \pm 2.4$ years. The survival curves and the cardiovascular fatal event-free rates are presented in Figures 3 and 4.

Thirty-one patients from the RVA group died as opposed to 24 in the RVOT group (HR, 0.96; 95\% CI, 0.57-1.65; $\mathrm{P}=0.89$ ). There were 10 cardiovascular deaths in the RVA group and 12 in the RVOT group (HR, 1.04; 95CI, 0.452.41; $\mathrm{P}=0.93)$. The groups were well matched, however multivariate analysis was performed to control for the potential biases. Adjusted analyses had a minimal influence on the estimate of the effect of the treatment on survival (Table 2). In the univariate analysis male gender, age at 
implantation, heart failure symptoms of NYHA III and LVEDD were significant risk factors of all-cause mortality. In the multivariate analysis only male gender and age at implantation remained significant predictors of all-cause death. In the RVOT group the HR of all-cause mortality, adjusted for age, gender, arterial hypertension, NYHA class and LVEDD was: HR, $1.41 ; 95 \% \mathrm{CI}, 0.76-2.64 ; \mathrm{P}=0.28$.

In relation to cardiovascular mortality male gender, age at implantation, NYHA III class, LVEDD and LVEF were significant risk factors in the univariate analysis. In the multivariate analysis male gender, age at implantation and NYHA III class remained significant predictors of cardiovascular death. The HR, adjusted for age, gender, atrial fibrillation, NYHA class and LVEDD, of cardiovascular mortality in the RVOT group was: HR, 1.66; 95\%CI, 0.614.52; $\mathrm{P}=0.32$. No sudden cardiac deaths occurred in our study population. There were no differences between RVA and RVOT group in fatal rates related to heart failure. There was 1 death due to chronic heart failure in the RVOT group and 2 in the RVA group. The vast majority of cardiovascular deaths were related to coronary artery disease $(6$ out of 12 deaths in the RVOT and 4 out of 10 in the RVA group). Other deaths were predominantly due to stroke.

Similarly, according to the on-treatment analysis, no differences were observed with regard to the all-cause (HR, $0.9 ; 95 \% \mathrm{CI}, 0.52-1.53 ; \mathrm{P}=0.69)$ or cardio-vascular death (HR, 0.70; 95\%CI 0.29-1.67; $\mathrm{P}=0.42$ ).

\section{Discussion}

In the present study we demonstrate effects of pacing at different RV sites on the total and cardiovascular mortality of patients with conventional antibradycardia indications, who presented with normal LV systolic function at the time of pacemaker implantation. This is the first prospective randomized study comparing the outcome of patients with preserved LV systolic function paced from the RVOT or RVA after a 10-year follow-up period. The results indicate that pacing at the RVOT provides no advantage over RVA in terms of survival.

Since cardiac pacing was introduced, the RVA has been used as the traditional site for ventricular lead placement. However, animal data and recent findings in humans have raised questions regarding RVA pacing, as it changes the sequence and synchrony of ventricular activation and leads to a decrease in LV function. ${ }^{9-11}$ These observations have driven the search for alternative ventricular pacing sites that might cause less electrical and contractile dyssynchrony. ${ }^{12}$ The preservation of LV function is especially important in patients with bradycardia who need long-term continuous ventricular support. Until now, however, there has been a search, still ongoing, for the most favorable pacing site within the RV.

During ventricular pacing, electrical activation spreads through the myocardium approximately four times slower than through the specialized conduction system. This slow conduction leads to electrical and contractile dyssynchrony between the early- and late-activated areas. ${ }^{11}$ Early-activated myocytes contract during early-systole whereas LV pressure is still low, thereby stretching the late-activated myocytes. When late-activated myocytes are excited, they contract powerfully due to their early-systolic stretch, resulting in systolic stretching of the early-activated myocytes. Thus, ventricular pacing produces electrical dyssynchrony, which in turn leads to pronounced mechanical dyssyn- chrony. Dyscoordinate contraction results in reduction in LV pump function and induces asymmetric myocardial remodeling, as late-activated myocytes become hypertrophied in response to increased regional loading conditions. It is of importance that, regardless of the exact pacing site, ventricular pacing always results in electrical dyssynchrony as compared to normal activation, although the degree of this dyssynchrony might vary between different ventricular pacing sites. The most favorable, namely His-bundle pacing, provides synchronous and unchanged electrical activation and unchanged LV pump function, but the implantation procedure is cumbersome. ${ }^{13}$ Unfortunately, pacing in the proximity of the His-bundle or bundle branches with the lead implanted at the RVOT area does not reproducibly achieve electrical activation of the conduction system. However, some pacing sites located outside the specialized conduction system, might provide better hemodynamics than others. It is thus of clinical importance to search for a ventricular pacing site that ensures the most favourable balance between the sequence of electrical activation and contraction dyssynchrony.

The RVOT area has been the most widely investigated among 'alternative' ventricular pacing sites. Since the first randomized trial in 1991 of Barin et al, who randomized 33 patients to RVA or RVOT pacing and showed comparable electrical performance and complication rate during followup, the RVOT area has paved the way for further clinical studies on non-apical ventricular pacing sites. ${ }^{14}$ The design of our study was proposed in 1995, when there was growing interest in the RV selective pacing sites and very strong believe in the superiority of RVOT over RVA pacing. The first patients were randomized at the end of 1995 and the last ones in 1997. Initially we thought we would be able to demonstrate the superiority of RVOT pacing on the basis of clinical and echocardiographic findings. However, we soon realized that the results had not provided evidence in favor of either pacing site. We were unable to demonstrate any advantage of RVOT over RVA pacing on the LV diastolic function during the 6 months follow-up. ${ }^{15}$ Data from the literature on the RVOT vs RVA debate are still conflicting, which might be attributed to the inhomogeneity of the studies performed in different patients populations, the small cohorts studied, the differing protocols used and the lack of accepted definitions of RV lead position.,5, $, 12,16-18$

In 2003, de Cock et al published a quantitative review that pooled the data of several small cohorts in which multiple outcome measures were compared during RVA and RVOT pacing. ${ }^{4}$ Using Cochrane search strategies, the authors reviewed the literature between 1984 and 2000 and included studies on pacing for conventional antibradycardia indications. These had to be prospective and to have reported measurements of LV systolic function. Eventually, from 17 studies identified, the authors included 9 on overall 227 patients. In spite of the fact that the overall result was in favor of the RVOT pacing, only 3 out of the 9 studies provided evidence that this area is superior to the RVA, whereas 6 did not. Most of the studies included had assessed the acute effect of RVOT in comparison to the RVA pacing, and, finally, the overall result was dominated by 1 acute study on a relatively large cohort of 89 patients, by Giudici et al. ${ }^{19}$ Moreover, in this study the proportion of superior results from RVOT pacing ( 85 out of 89 patients) and the relative improvement (on average $21 \%$ of the basal cardiac index during RVA pacing) were unusually high compared to many other reports. This makes the main basis of the 
pooled effect of the meta-analysis of de Cock et al somewhat questionable. 4

Data comparing RVOT to RVA pacing in chronically implanted patients also provided inconsistent results. Victor et al conducted one of the first studies evaluating functional and hemodynamic effects of RVA and RVOT pacing in 16 patients and found no differences after 3 months of pacing. ${ }^{5}$ This study was included in the meta-analysis of de Cock et al. ${ }^{4}$ Investigators of the ROVA study ${ }^{6}$ made a clinical evaluation in a randomized cross-over study after a 3 months pacing period, in a large group of 103 pacemaker recipients with congestive heart failure and atrial fibrillation. The authors found no differences between the RVA and RVOT group in the quality of life scores, NYHA class, 6 min walking distance, LV function or the degree of mitral regurgitation.

There are only two long-term studies to date comparing clinical effects of RVOT and RVA pacing. ${ }^{7,16}$ In 2002, Tse et al in a study of 24 patients randomized to RVOT or RVA lead placement, demonstrated that RVOT pacing prevented deleterious effects of RVA pacing on myocardial perfusion and function in the 18-month observation period. ${ }^{7}$ Our group reported similar results, ${ }^{16}$ after comparison of the clinical effects in 27 patients paced at the RVOT or RVA for 7 years. It was shown that with the lead placed at the RVOT the ventricular remodeling caused by permanent RV pacing could be slowed down, as LVEF was preserved in the RVOT group, while decreasing in the RVA group. However, the left atrial enlargement and progression of the LV wall hypertrophy in the RVOT group was noticed, which was not found in the RVA group. Both studies demonstrated that RVOT pacing at its best could diminish the deleterious effects of RVA pacing on LV function, but could certainly not improve LV function.

It should be underlined that the pacing site in our study was the RVOT and not the mid-septum, which according to Durrer et al is the region of the earliest ventricular activation. ${ }^{20}$ Pacing from the RV mid-septum could provide a more physiological activation pattern. ${ }^{21}$ Victor et al compared chronic mid-septal and RVA pacing in 28 patients with permanent atrial fibrillation, in whom ablation of the AV junction was performed, followed by implantation of a DDDR pacemaker connected to 2 ventricular leads. ${ }^{22}$ Septal pacing was associated with shorter QRS and in contrast to RVA pacing preserved LVEF. Muto et al provided similar observations on a much larger group of patients. ${ }^{23}$ The authors followed up 273 patients with permanent atrial fibrillation and LVEF $<30 \%$ randomized to RV mid-septal or apical pacing for a period of 18 months, and reported on improved LVEF and quality of life associated with midseptal pacing in contrast to RVA pacing.

It needs to be emphasized that the study presented has been the largest published so far on the long-term effects of alternative RV pacing sites. In spite of the use of passive fixation leads, the RV lead location remained stable during the long-term follow-up as comparison of the discharge ECGs with the last available ECG in a particular patient revealed unchanged QRS axis and morphology. Schwaab et al in a group of 120 patients observed for a mean period of 14 months, also demonstrated that passive fixation leads could easily be implanted in the RVOT septum with no increased risk of dislocation, and with maintenance of appropriate pacing parameters. ${ }^{24}$

The 10-year survival analysis revealed no additional benefit of RVOT over RVA pacing regarding the all-cause and cardiovascular mortality neither according to the intention-to-treat or the on-treatment analysis. The demographic and clinical data of patients from the 2 groups did not differ at the time of implantation. Of the different factors studied, the ventricular pacing site remained an insignificant risk factor of either all-cause or cardiovascular mortality, both in the univariate or multivariate analysis.

A possible explanation of our results is that the RVOT does not provide a consistent beneficial hemodynamic effect over the RVA pacing, as the RV pacing site that leads to the least reduction of pump function varies between patients. In the study of Lieberman et al the authors assessed the influence of several RV pacing sites (apex, free wall and septum) on global cardiac performance, and found that $\mathrm{RV}$ pacing worsens $\mathrm{LV}$ function unless the pacing site is optimized. ${ }^{25}$ There were substantial inter-individual variations in the optimal RV pacing site, and no consistent site was superior to others. These observations support results from our study. The RVOT in general cannot be regarded superior to the RVA, and the pacing site should be individually optimized. With respect to the contraction synchrony, evaluation on tissue Doppler echocardiography seems valuable, although this approach was not applied in our study. ${ }^{26}$

In our opinion, the QRS duration during pacing at a given site can be regarded only as a rough marker of LV hemodynamics. Schwaab et al ${ }^{17}$ showed that a reduction in QRS duration by RVOT pacing is significantly correlated with homogenization of LV contraction and improved systolic function, however other studies have shown lack of correlation between QRS duration and cardiac function during ventricular pacing. ${ }^{10}$ Liebermann et al also found no correlation between the QRS width and the hemodynamic effect of a given RV pacing site. ${ }^{25}$ In our study ventricular lead position during the implantation procedure was verified only on the basis of fluoroscopy and the QRS width compared several days after implantation did not differ between groups. Because QRS duration represents biventricular electrical activation, changes in determinants of cardiac function, such as the sequence of activation and intraventricular synchrony, are not necessarily translated into changes in the length of the QRS.

\section{Study Limitations}

We have to underline that the ventricular lead position in our study was the RVOT and not RV mid-septum or the proximity of the His-bundle which, according to the results of studies designed later than ours, can provide a more physiological propagation of electrical conduction. ${ }^{13}$ The position of the electrode in the RVOT was based only on fluoroscopic images during the implantation procedure, however this was the standard approach in the daily practice at the time of the randomization process. The AV delay optimization was not performed on a routine basis, and modified only during the follow-up if important AV dyssynchrony was noticed during the echo-Doppler examination. However, the mean AV delay during the last control visit was comparable to the mean optimal value reported in patients with preserved LV function from other studies. ${ }^{27}$

\section{Conclusions}

The results of our study indicate that there were no differences in survival in patients with standard pacing indications and preserved LV function with septal RVOT or apical RV lead placement during the long-term follow-up. 
It cannot be recommended to implant the RV pacing lead in the RVOT instead of the RVA in the general population. Only individual positioning of the RV pacing lead aimed at providing LV contraction synchrony might result in better outcome.

\section{Disclosure}

The authors declare no conflict of interests.

\section{References}

1. Manolis AS. The deleterious consequences of right ventricular apical pacing: Time to seek alternate site pacing. Pacing Clin Electrophysiol 2006; 29: $298-315$

2. Vanagt WY, Prinzen FW, Delhaas T. Physiology of cardiac pacing in children. Pacing Clin Electrophysiol 2008; 31(Suppl 1): S24-S27.

3. Seo Y, Ishizu T, Nakajima H, Sekiguchi Y, Watanabe S, Aonuma K. Clinical utility of 3-dimensional echocardiography in the evaluation of tricuspid regurgitation caused by pacemaker leads. Circ J 2008; 72: $1465-1470$.

4. de Cock CC, Giudici MC, Twisk JW. Comparison of the haemodynamic effects of right ventricular outflow-tract pacing with right ventricular apex pacing: A quantitative review. Europace 2003; 5: 275-278.

5. Victor F, Leclercq C, Mabo P, Pavin D, Deviller A, de Place C, et al. Optimal right ventricular pacing site in chronically implanted patients: A prospective randomized crossover comparison of apical and outflow tract pacing. J Am Coll Cardiol 1999; 33: 311-316.

6. Stambler BS, Ellenbogen K, Zhang X, Porter TR, Xie F, Malik R, et al. Right ventricular outflow versus apical pacing in pacemaker patients with congestive heart failure and atrial fibrillation. J Cardiovasc Electrophysiol 2003; 14: 1180-1186.

7. Tse HF, Yu C, Wong KK, Tsang V, Leung YL, Ho WY, et al. Functional abnormalities in patients with permanent right ventricular pacing: The effect of sites of electrical stimulation. J Am Coll Cardiol 2002; 40: $1451-1458$.

8. Cox DR. Regression models and life-tables. JR Stat Soc [B] 1972; 34: $187-220$.

9. Prinzen FW, Peschar M. Relation between the pacing induced sequence of activation and left ventricular pump function in animals. Pacing Clin Electrophysiol 2002; 25(4 Pt 1): 484-498.

10. Tse HF, Lau CP. Long-term effect of right ventricular pacing on myocardial perfusion and function. J Am Coll Cardiol 1997; 29: 744-749.

11. Tantengco MV, Thomas RL, Karpawich PP. Left ventricular dysfunction after long-term right ventricular apical pacing in the young. J Am Coll Cardiol 2001; 37: 2093-2100.

12. Kiuchi K, Yoshida A, Fukuzawa K, Takano T, Kanda G, Takami K, et al. Identification of the right ventricular pacing site for cardiac resynchronization therapy (CRT) guided by electroanatomical mapping (CARTO). Circ J 2007; 71: 1599-1605.

13. Deshmukh P, Casavant DA, Romanyshyn M, Anderson K. Permanent, direct His-bundle pacing: A novel approach to cardiac pacing in patients with normal His-Purkinje activation. Circulation 2000; 101: $869-877$.
14. Barin ES, Jones SM, Ward DE, Camm AJ, Nathan AW. The right ventricular outflow tract as an alternative permanent pacing site: Longterm follow-up. Pacing Clin Electrophysiol 1991; 14: 3-6.

15. Baczynska AM, Wilczek R, Staniewicz J, Dabrowska-Kugacka A, Swiatecka G. Diastolic function in patients with right ventricular outflow tract vs right ventricular apex pacing. In: Vardas PE, editor. Europace ' 97 Free Papers. Athens: Monduzzi Editore International Proceedings Division; 1997; 701-704.

16. Lewicka-Nowak E, Dabrowska-Kugacka A, Tybura S, KrzyminskaStasiuk E, Wilczek R, Staniewicz J, et al. Right ventricular apex versus right ventricular outflow tract pacing: Prospective, randomized, long-term clinical and echocardiographic evaluation. Kardiol Pol 2006; 64: 1082-1091.

17. Schwaab B, Fröhlig G, Alexander C, Kindermann M, Hellwig N, Schwerdt $\mathrm{H}$, et al. Influence of right ventricular stimulation site on left ventricular function in atrial synchronous ventricular pacing. $J \mathrm{Am}$ Coll Cardiol 1999; 33: 317-323.

18. de Cock CC, Meyer A, Kamp O, Visser CA. Hemodynamic benefits of right ventricular outflow tract pacing: Comparison with right ventricular apex pacing. Pacing Clin Electrophysiol 1998; 21: 536541.

19. Giudici MC, Thornburg GA, Buck DL, Coyne EP, Walton MC, Paul DL, et al. Comparison of right ventricular outflow tract and apical lead permanent pacing on cardiac output. Am J Cardiol 1997; 79: 209-212.

20. Durrer D, Van Dam RT, Freud GE, Janse MJ, Meijler FL, Arzbaecher RC. Total excitation of the isolated human heart. Circulation 1970; 41: 899.

21. Peschar M, de Swart H, Michels KJ, Reneman RS, Prinzen FW. Left ventricular septal and apex pacing for optimal pump function in canine hearts. J Am Coll Cardiol 2003; 41: 1218-1226.

22. Victor F, Mabo P, Mansour H, Pavin D, Kabalu G, de Place C, et al. A randomized comparison of permanent septal versus apical right ventricular pacing: Short-term results. J Cardiovasc Electrophysiol 2006; 17: $238-242$.

23. Muto C, Ottaviano L, Canciello M, Carreras G, Calvanese R, Ascione $\mathrm{L}$, et al. Effect of pacing the right ventricular mid-septum tract in patients with permanent atrial fibrillation and low ejection fraction. J Cardiovasc Electrophysiol 2007; 18: 1032-1036.

24. Schwaab B, Kindermann M, Fröhlig G, Berg M, Kusch O, Schieffer H. Septal lead implantation for the reduction of paced QRS duration using passive-fixation leads. Pacing Clin Electrophysiol 2001; 24: $28-33$.

25. Lieberman R, Padeletti L, Schreuder J, Jackson K, Michelucci A, Colella A, et al. Ventricular pacing lead location alters systemic hemodynamics and left ventricular function in patients with and without reduced ejection fraction. J Am Coll Cardiol 2006; 48: $1634-1641$.

26. Takamatsu H, Tada H, Okaniwa H, Toide H, Maruyama H, Higuchi $\mathrm{R}$, et al. Right bundle branch block and impaired left ventricular function as evidence of a left ventricular conduction delay. Circ J 2008; 72: $120-126$.

27. Miki Y, Ishikawa T, Matsushita K, Yamakawa Y, Matsumoto K, Sumita S, et al. Novel method of predicting the optimal atrioventricular delay in patients with complete AV block, normal left ventricular function and an implanted DDD pacemaker. Circ J 2009; 73: 654657. 